\title{
Proton Form Factors and Related Processes in BaBar by ISR
}

\author{
Rinaldo Baldini Ferroli representing the BaBar collaboration \\ Centro Studi e Ricerche Enrico Fermi, Roma, Italy \\ INFN, Laboratori Nazionali di Frascati, Italy \\ baldini@centrofermi.it
}

\begin{abstract}
BaBar has measured with unprecedented accuracy $e^{+} e^{-} \rightarrow p \bar{p}$ from the threshold up to $Q_{p \bar{p}}^{2} \sim 20 \mathrm{GeV}^{2} / \mathrm{c}^{4}$, finding out an unexpected cross section, with plateaux and drops. In particular it is well established a sharp drop near threshold, where evidence for structures in multihadronic channels has also been found. Other unexpected and spectacular features of the Nucleon form factors are reminded, the behaviour of spacelike $G_{E}^{p} / G_{M}^{p}$ and the neutron time-like form factors.
\end{abstract}

\section{Introduction and space-like Nucleon form factors}

The Nucleon form factors (FF) are among the very first topics have been studied in subnuclear physics. In spite of many decades of experimental investigation Nucleon FF have recently shown rather unexpected and spectacular features, namely:

- in $e^{-} p \rightarrow e^{-} p$ scattering, i.e. space-like exchanged $Q^{2}$ (in the following assumed to be negative), the ratio $\left|G_{E}^{p} / G_{M}^{p}\right|$ falls down approaching zero at $Q^{2} \sim 8 \mathrm{GeV}^{2}$ [1], despite that in all textbooks for about forty years it was assumed to be a constant and despite early, foresighted predictions $[2,3]$;

- in $e^{+} e^{-} \rightarrow p \bar{p}$ annihilation, i.e. time-like (positive) exchanged $Q^{2}$, BaBar has shown that the FF have a step-like behaviour [4], despite that everybody had assumed a smoothed one;

- in particular in $e^{+} e^{-} \rightarrow p \bar{p}$ a sharp drop just above threshold [3,4] strongly suggests a narrow resonance at threshold, despite some time ago the existence of a baryonium had been dismissed;

- the $e^{+} e^{-} \rightarrow n \bar{n}$ cross section, at least according to the only measurement performed until now [5], has been found about an order of magnitude larger than expected.

In the following the recent space-like results on Nucleon FF are summarized and the brand new time-like results, obtained by BaBar by means of Initial State Radiation (ISR), are described more in detail. Let remind that the FF definition is somewhat tricky: they are defined as those factors, which account for the hadronic 
structure, in the one photon exchange amplitude [6]. In some cases two photons exchange amplitudes are important, they have to be evaluated and subtracted, affecting in a different way different observables.

In the elastic electron-Nucleon scattering, i.e. space-like exchanged $Q^{2}$, magnetic $G_{M}\left(Q^{2}\right)$ and electric $G_{E}\left(Q^{2}\right)$ FF enter the spin flip and non spin flip amplitude, as defined in the Nucleon Breit frame. Because of their definition magnetic and electric FF do not interfere.

Proton and neutron space-like magnetic form factors are quite established as well as their overall behaviour is fairly described by a dipole formula. The field theory expectation is that the FF of a hadron, made of $n$ constituents, should scale asymptotically with $Q^{2}$ as $Q^{-2(n-1)}$ [7] and magnetic form factors scale as $Q^{-4}$, in agreement with PQCD and quark model (assuming 3 valence quarks in the Nucleon). This is not the case of the proton space-like electric form factor. Actually it is not a straightforward measurement. As soon as $Q^{2} \sim M_{N}^{2}$, because of the $\mu_{N}^{2} \cdot Q^{2} / M_{N}^{2}$ factor the magnetic contribution is dominant, the electric contribution is at a few percent level and two photons exchange contribution might play an important role, heavily affecting the Born term. The ratio between electric and magnetic FF can also be obtained by looking to the ratio between transverse and parallel polarization of the outgoing Nucleon. This kind of measurement is not affected by the aforementioned factor and it is less sensitive to radiative corrections. In Fig.1, the ratio $G_{E}^{p}\left(Q^{2}\right) / G_{M}^{p}\left(Q^{2}\right)$ is plotted, as obtained mostly at JLAB few years ago [1], according to the polarization ratio, compared to the almost constant expectation according to the angular distribution by means of the Rosenbluth method, as obtained at SLAC [8] and at JLAB too. The two sets of measurements disagree completely and JLAB polarization data destroy the "scaling law" between electric and magnetic FF, considered like a dogma for many decades. As a consequence, at $Q_{p \bar{p}}^{2} \sim 8 \mathrm{GeV}^{2}$ a zero of $G_{E}^{p}\left(Q_{p \bar{p}}^{2}\right)$ (followed by a negative tail reaching the value -1 asymptotically [9]) is foreseen and soon this expectation will be tested at JLAB. Two photons contributions are difficult to evaluate, depending on the Nucleon structure too. However present evaluations [10] show that sign and order of magnitude both are in the right direction and it is very likely they are responsible of this spectacular discrepancy. A measurement by means of a positron beam will disentangle the question of two photons contribution [11].

It might be worthwhile to remind that many decades ago T. Massam and A. Zichichi formulated a model of the Nucleon [2], forerunner of the present soliton models (assuming an extended source of vector mesons with a $1 / M_{N}$ radius), that foresaw for the proton a fast drop with $Q_{p \bar{p}}^{2}$ of $G_{E}^{p}\left(Q_{p \bar{p}}^{2}\right) / G_{M}^{p}\left(Q_{p \bar{p}}^{2}\right)$, as shown in Fig.1. Adding a point-like source contribution F. Iachello, A.D. Jackson and A. Lande [3] improved this model and their prediction is in astonishing agreement with the JLAB data, as shown in Fig.1. PQCD may also predict a similar result, assuming important NLO corrections. The two calculations do not behave in the same way once extrapolated to time-like $Q_{p \bar{p}}^{2}$ (in a somewhat plausible but not rigorous way). 


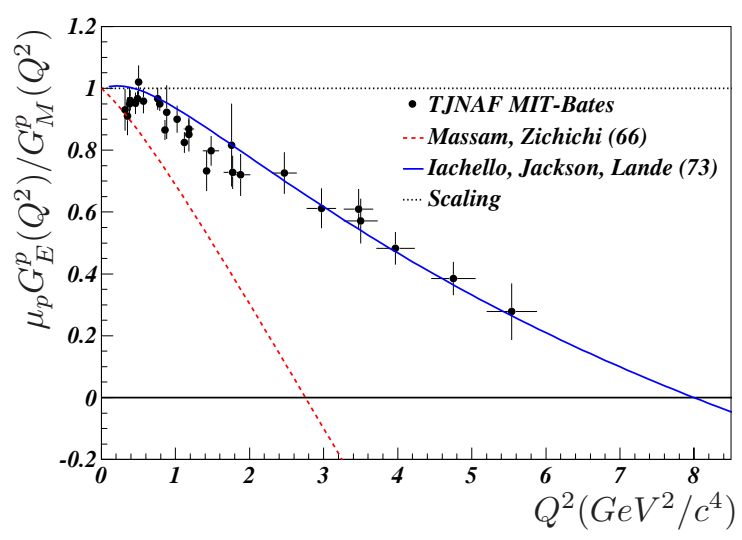

Figure 1. $\mu_{p} G_{E}^{p}\left(Q^{2}\right) / G_{M}^{p}\left(Q^{2}\right)$, as achieved by means of transverse and longitudinal outgoing proton polarization. The former dogma, the scaling law $\mu_{p} G_{E}^{p}\left(Q^{2}\right) / G_{M}^{p}\left(Q^{2}\right) \sim 1$, is also pointed out. The dashed line is a prediction made about forty years ago [2], modelling the Nucleon like a soliton, and the solid line is the impressive prediction made some years later [3], adding a point-like coupling.

\section{Time-like Nucleon FF,}

BaBar results on $e^{+} e^{-} \rightarrow p \bar{p}$ and related processes

Analyticity is supposed to connect space-like FF, that are real quantities according to e.m. current conservation, and time-like FF, that have an imaginary part above the first isovector/isoscalar inelastic thresholds. In $e^{+} e^{-}$annihilation into $N \bar{N}$, i.e. time-like exchanged $Q^{2}$, two $\mathrm{FF}$ are also defined. This time they refer to the total Nucleon helicity, as defined in the c.m. frame. Particle-antiparticle relationship relates the total helicity equal to 0 form factor to $G_{M}$ and the total helicity equal to 1 form factor to $G_{E}$. Two other FF, $F_{1}\left(Q^{2}\right)$ and $F_{2}\left(Q^{2}\right)$ (Dirac and Pauli FF), can also be defined. Their relationship with the e.m. current $J^{\mu}$ and the anomalous magnetic moment $k$ is more transparent, namely: $J^{\mu}=F_{1}\left(Q^{2}\right) \gamma^{\mu}+\frac{k}{2 M} F_{2}\left(Q^{2}\right) i \sigma^{\mu \nu} q_{\nu}$ and

$$
G_{E}=F_{1}+\frac{Q^{2}}{4 M_{N}^{2}} F_{2}, \quad G_{M}=F_{1}+F_{2} .
$$

$F_{1}$ and $F_{2}$ as well as $G_{E}$ and $G_{M}$ analyticity demands that it is

$$
G_{E}\left(4 M_{N}^{2}\right)=G_{M}\left(4 M_{N}^{2}\right) .
$$

The same result is achieved if at threshold there is the $\mathrm{S}$ wave only.

The standard explanation of the $G_{E} / G_{M}$ drop for space-like $Q^{2}$ is because of a negative interference between $F_{1}$ and $F_{2}$. Therefore it should become a positive interference for time-like $Q^{2}$ and an increasing ratio $G_{E} / G_{M}$ above threshold is guessed. For time-like $Q^{2}, F_{1}$ and $F_{2}$ are complex functions and to get their relative phase, which has to vanish exactly at threshold, polarization tools are needed.

Dispersion relations can be applied to get $\mathrm{FF}$ in the whole unphysical region $\left(0<Q^{2}<4 M_{N}^{2}\right)$ as it is illustrated in ref. [12] in the case of $G_{M}^{p}$.

BaBar has recently measured [4] with unprecedented accuracy the cross section $\sigma\left(e^{+} e^{-} \rightarrow p \bar{p} \gamma\right)$ from the threshold up to a $p \bar{p}$ c.m. total energy squared $Q_{p \bar{p}}^{2} \sim$ $20 \mathrm{GeV}^{2} / \mathrm{c}^{4}$ by means of ISR. In fact it has been shown [13] that at a $m_{e} / E_{e}$ 


$$
\frac{d \sigma}{d \Omega_{\gamma} d E_{\gamma}}\left(e^{+} e^{-} \rightarrow p \bar{p} \gamma\right)=P\left(s, E_{\gamma}, \Omega_{\gamma}\right) \cdot \sigma\left(Q_{p \bar{p}}^{2}\right)
$$

where $s$ is the $e^{+} e^{-}$total c.m. energy squared, $E_{\gamma}$ and $\Omega_{\gamma}$ are energy and angles of the ISR photon in the $e^{+} e^{-}$c.m. frame, $P$ is the density function for ISR emission, computed according to $\mathrm{QED}$, and $\sigma\left(Q_{p \bar{p}}^{2}\right)$ is the $e^{+} e^{-} \rightarrow p \bar{p}$ cross section at the $p \bar{p}$ c.m. energy squared $Q_{p \bar{p}}^{2}$. In the Born approximation it is given by [6]:

$$
\frac{d \sigma\left(e^{+} e^{-} \rightarrow p \bar{p}\right)}{d \cos \theta}=\frac{\pi \alpha^{2} \beta C}{2 Q_{p \bar{p}}^{2}}\left[\left(1+\cos ^{2} \theta\right)\left|G_{M}^{p}\left(Q_{p \bar{p}}^{2}\right)\right|^{2}+\frac{4 M_{N}^{2}}{Q^{2}} \sin ^{2} \theta\left|G_{E}^{p}\left(Q_{p \bar{p}}^{2}\right)\right|^{2}\right] .(4)
$$

All quantities are evaluated in the $p \bar{p}$ c.m. frame: $\theta$ is the azimuthal proton emission angle, $\beta$ is the proton velocity, $C$ is a factor introduced in the final state distorted wave approximation to take into account the Coulomb interaction [14], $G_{E}^{p}$ and $\left|G_{M}^{p}\right|$ the analytical time-like continuation of the corresponding space-like FF. As mentioned already at $Q_{p \bar{p}}^{2}=4 M_{p}^{2}$ it is expected $G_{E}^{p} / G_{M}^{p}=1$. So far this expectation has been extended to the whole $Q_{p \bar{p}}^{2}$ explored range [5,15-20], lacking high statistics measurements in particular concerning $G_{E}$. Actually what is quoted is $\left|G_{M}^{p}\right|$, also because at high $Q_{p \bar{p}}^{2}$ its contribution is the dominant one. Concerning time-like $Q_{p \bar{p}}^{2}$ the first [15] and the most relevant measurements at low [16] and at high $Q_{p \bar{p}}^{2}$ [17], previous to BaBar, have been performed by means of the inverse process $p \bar{p} \rightarrow e^{+} e^{-}$. Under the aforementioned hypothesis concerning $\left|G_{M}^{p}\right|$ they have shown a very steep increase approaching the threshold and a $1 / Q_{p \bar{p}}^{4}$ overall behaviour, quite earlier than asymptotically expected according to PQCD [21] as in the space-like region.

In the following ISR events have been selected by asking the ISR photon is detected, to get rid of the non ISR multihadronic background. A rather large fraction of events is lost in this way, however in this case BaBar has the advantage over a conventional c.m. $e^{+} e^{-}$collider that the cross section can be measured even at threshold, with a $\sim 1 \mathrm{MeV} / \mathrm{c}^{2} Q_{p \bar{p}}$ invariant mass resolution and with almost full $p \bar{p}$ angular coverage.

The B-factory PEP II $\left(9 \mathrm{GeV} / \mathrm{c}^{2} e^{-}\right.$colliding with $\left.3.1 \mathrm{GeV} / \mathrm{c}^{2} e^{+}\right)$and the BaBar detector have been described in detail several times [22]. For the present purposes charged particle identification is mostly done by means of an internally reflecting ring imaging Cherenkov (DIRC). The Monte Carlo (MC) event generator is based on the code described in [23]. Extra ISR soft photons are generated according to the structure function method [24].

At present a data sample related to $232 \mathrm{fb}^{-1}$ total integrated luminosity has been analyzed. Event selection has required an energetic photon and two opposite sign charged tracks, originated from the interaction point with a polar angle within the DIRC acceptance. To get rid of the huge ISR $\pi^{+} \pi^{-} \gamma, \mu \mu \gamma, K^{+} K^{-} \gamma$ background both charged tracks are required to be well identified as proton candidates by means 

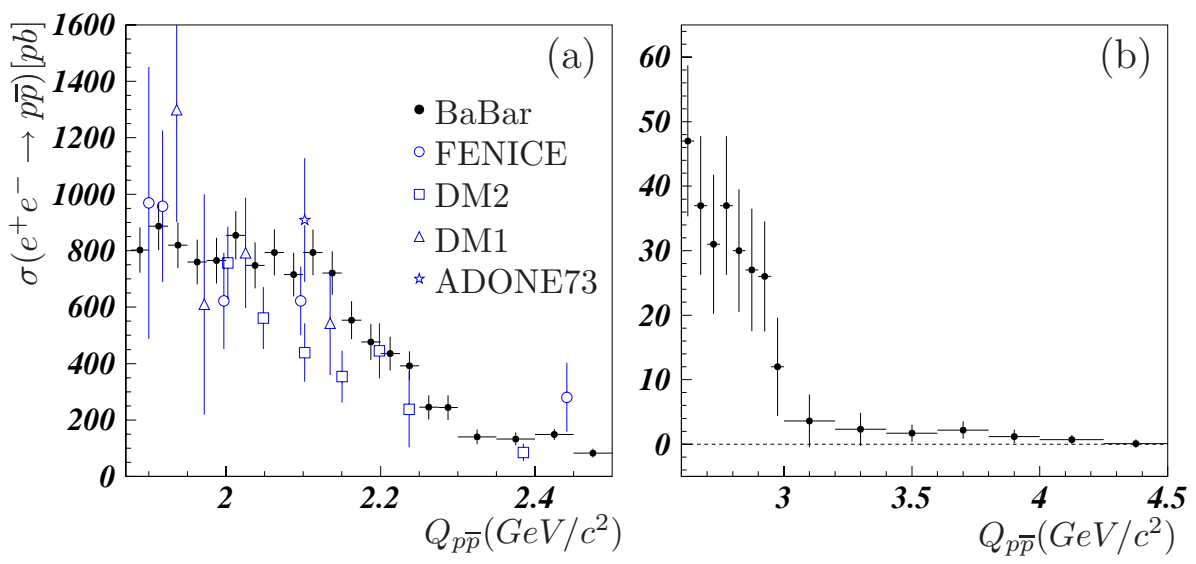

Figure 2. BaBar $e^{+} e^{-} \rightarrow p \bar{p}$ cross section in comparison with previous experiments: FENICE [5], DM2 [20], DM1 [19] and ADONE73 [18].

of a tight cut, leading to a loss of about $30 \%$ of signal events. As anticipated the ISR photon detection is required, but photon information have not been used in the following analysis. A $1 \mathrm{C}$ kinematical fit is performed adding further tight cuts, that is to be compatible only with proton masses hypothesis, leading to a further loss of about $25 \%$. The overall detection efficiency is about $18 \%$ with a mild dependence on $Q_{p \bar{p}}$. In the surviving events there is no evidence of a peak at the $\rho$ mass or a peak at the $\Phi$ mass in a pion or kaon pair masses hypothesis and the estimated remaining contamination is negligible. Angular and energy photon distributions are consistent with the ISR expectation. Final state radiation is expected to be very small and there is no interference term, due to the different charge parity. The most important and subtle source of background is the process $e^{+} e^{-} \rightarrow p \bar{p} \pi^{0}$, where easily a soft photon is lost or the two photons are merged and not disentangled by the pattern recognition. Since there are no experimental information on this process, events $e^{+} e^{-} \rightarrow p \bar{p} \pi^{0}$ with a fully reconstructed $\pi^{0}$ are identified and the contamination in the selected sample of $e^{+} e^{-} \rightarrow p \bar{p}$ candidate events is evaluated according to the MC expectation. These estimated background events are about $5 \%$ of the selected candidate events when $Q_{p \bar{p}}^{2}<6 \mathrm{GeV}^{2} / \mathrm{c}^{4}$ and become consistent with $100 \%$ above $Q_{p \bar{p}}^{2} \sim 20 \mathrm{GeV}^{2} / \mathrm{c}^{4}$.

The ISR luminosity, that has been integrated, depends on the $Q_{p \bar{p}}$ invariant mass bin width, varying from $\sim 0.5 p b^{-1}$ at $Q_{p \bar{p}} \sim 2 \mathrm{GeV} / \mathrm{c}^{2}$ up to $\sim 1 p b^{-1}$ at $Q_{p \bar{p}} \sim 3 \mathrm{GeV} / \mathrm{c}^{2}$ for a $10 \mathrm{MeV} / \mathrm{c}^{2}$ bin width. Radiative corrections have been evaluated according the structure function method. They do not include corrections due to vacuum polarization. Hence what is quoted here is the so called "dressed" cross section. The invariant mass resolution has been unfolded, however the chosen bin widths exceed the resolution. With all these ingredients the calculated cross section $\sigma\left(e^{+} e^{-} \rightarrow p \bar{p}\right)$ is shown in Fig.2, statistical and systematic errors are quoted, 
including the uncertainties in detection efficiency, integrated luminosity and radiative corrections. For comparison some previous measurements are also shown. The emerging cross section shape is an unexpected one: a flat plateau from the threshold up to $Q_{p \bar{p}}^{2} \sim 5 \mathrm{GeV}^{2} / \mathrm{c}^{4}$, followed by a drop and then by a second drop at $Q_{p \bar{p}}^{2} \sim 8.5 \mathrm{GeV}^{2} / c^{4}$. At present no simple explanation has been found concerning this behaviour.

The angular distributions have been fitted, according to eq.(4) to extract the ratio $\left|G_{E}^{p} / G_{M}^{p}\right|$. The fitted ratios are reported in the Fig.3 . BaBar results are not in agreement with the APPLE [16] results at LEAR, while they are in qualitative agreement with an expectation, updated, based on dispersion relations and on the JLAB space-like $\left|G_{E}^{p} / G_{M}^{p}\right|$ measurements [9]. There is no clear evidence for an asymmetry, i.e. two photons exchange contribution, but there is not enough statistics to draw any conclusion.

As previous experiments did, $\left|G_{M}\right|$ is evaluated from the total cross section as shown in Fig.4, under the hypothesis $G_{E}=G_{M}$, which is at odd with the aforementioned results by the way. The asymptotic $1 /\left(Q_{p \bar{p}}^{2}\right)^{2}$ behaviour found in $p \bar{p} \rightarrow e^{+} e^{-}$is well established as well as a very steep slope very near threshold.

The Nucleon FF and other recent results on sharp behaviours at low $p \bar{p}$ invariant mass have produced a "baryonium" [25] revival, that is a $N \bar{N}$ bound/resonant state, searched and lusted in vain for a long time. In particular BESII pointed out a $\mathrm{C}=+$ narrow state below the $p \bar{p}$ threshold, looking at $J / \psi \rightarrow \gamma p \bar{p}[26]$. In the FF case a vector baryonium is expected as a fairly narrow resonance, that should be seen also in other hadronic channels, even if a vanishing coupling to $e^{+} e^{-}$is foreseen. However such a resonance, mixing with a wide $q \bar{q}$ vector meson near by, should always make a dip in a $e^{+} e^{-}$multihadronic annihilation channel [27]. Actually dips near the $p \bar{p}$ threshold have been observed, for instance in $\sigma_{t o t}\left(e^{+} e^{-} \rightarrow\right.$ hadrons) [2] and in a peculiar channel, namely in $e^{+} e^{-} \rightarrow 3 \pi^{+} 3 \pi^{-}$by DM2 [28], in diffractive $3 \pi^{+} 3 \pi^{-}$photoproduction by E687 [29], and finally in $e^{+} e^{-} \rightarrow 3 \pi^{+} 3 \pi^{-}$ and $e^{+} e^{-} \rightarrow 2 \pi^{+} 2 \pi^{-} 2 \pi^{0}$ by BaBar by means of ISR [30]. However this dip in the $6 \pi$ channel is hardly compatible with a resonance below threshold and it has not been seen by Obelix in $n \bar{p}$ [31] (even if it was expected near the edge of their mass acceptance). Remarkably enough an interference pattern below and above threshold was predicted by dispersion relations, applied to get the magnetic form factor in the unphysical region, without using the data very near threshold [12].

Alternative interpretations of the proton form factor drop at threshold are $p \bar{p}$ final state interactions [32]. The $C$ factor in the $p \bar{p}$ cross section formula has a very steep slope too and is relevant only very near threshold, diverging as $1 / \beta$ so that the cross section should be finite at threshold. It has been introduced to get rid of the pointlike Coulomb interaction. However this receipt concerning Coulomb interaction has been questioned [33] and a better evaluation might affect the steep threshold behaviour of the FF. It may be worthwhile to remind that no Coulomb corrections are expected in the case of $e^{+} e^{-} \rightarrow n \bar{n}$. 


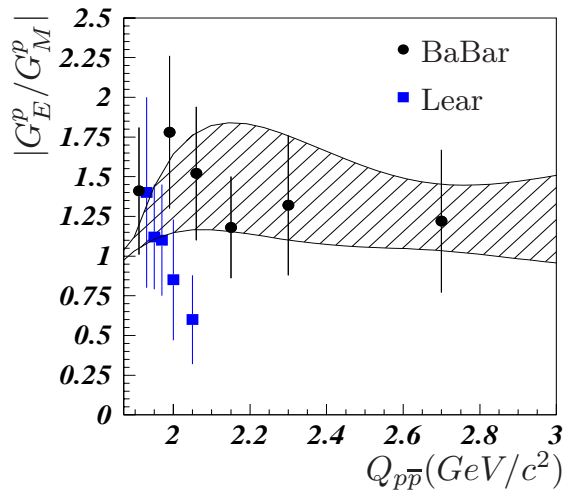

Figure 3. The measured ratio $\left|G_{E}^{p} / G_{M}^{p}\right|$ : BaBar, Lear data [16] and according to analyticity [9].



Figure 4. Proton magnetic form factor measurements near threshold, assuming $G_{M}^{p}=G_{E}^{p}$.

3. $e^{+} e^{-} \rightarrow n \bar{n}$

The $e^{+} e^{-} \rightarrow n \bar{n}$ cross section has been measured only once, 15 years ago, by the FENICE experiment at the old storage ring ADONE in Frascati [5]. The collected luminosity was very poor: $\sim 500 \mathrm{nb}^{-1}$ in the c.m. energy range $1.9<Q<2.55 \mathrm{GeV}$. The $e^{+} e^{-} \rightarrow p \bar{p}$ cross section as well as $\sigma_{t o t}\left(e^{+} e^{-} \rightarrow\right.$ hadrons $)$ and QED processes have also been measured. It has been found

$$
R_{N}=\frac{\sigma\left(e^{+} e^{-} \rightarrow n \bar{n}\right)}{\sigma\left(e^{+} e^{-} \rightarrow p \bar{p}\right)} \sim 2 .
$$

Assuming a leading quark in the Nucleon it is expected $R_{N} \sim\left(\frac{q_{d}}{q_{u}}\right)^{2}=0.25$. The same result is obtained by a calculation based on a dispersive approach and PQCD asymptotic behaviour [34]. In any PQCD calculation relying on valence quarks it is quite difficult to predict $R_{N}$ greater or equal to 1 , while soliton models expect $R_{N} \geq 1$ [35] and some VMD based models predicted even $R_{N}>1$ [36]. The average angular distribution is consistent with $G_{E}^{n}$ much lower than $G_{M}^{n}$ as in the space-like region [5]. The neutron magnetic form factor is shown in Fig.5, as achieved by FENICE and by DM2 also. It has to be stressed that the measurement of $e^{+} e^{-} \rightarrow n \bar{n}$ has to be redone with much higher collected luminosity. Unfortunately it is a very difficult task, if not impossible, by means of ISR in BaBar.

\section{Conclusions}

In the near future the new $e^{+} e^{-}$symmetric storage ring VEPP2000 in Novosibirsk should collect data up to a c.m. total energy of $2 \mathrm{GeV}$ at most. However, in the near threshold region, to get rid of the background without boosting the c.m. will not be a simple job. BEPC2, the new $\tau /$ Charm Factory in Beijing and, possibly DANAE, a proposed DA $\Phi$ NE upgrade in energy and in luminosity in Frascati, should have a wider available c.m. energy range. In conclusion Nucleon FF have recently shown 


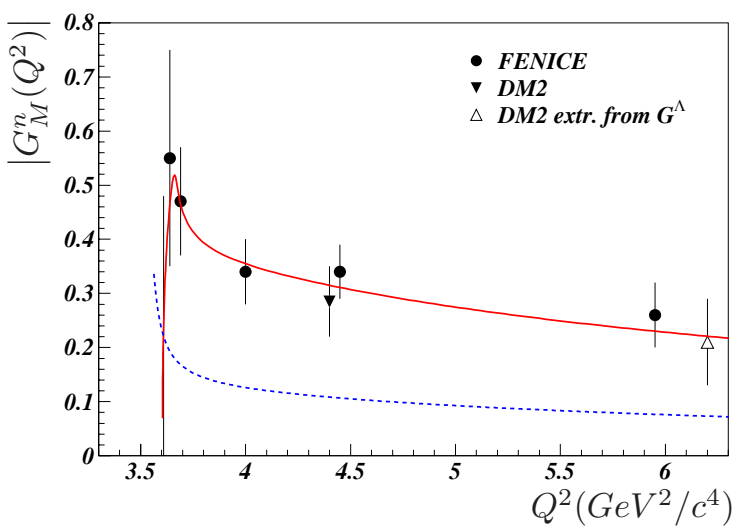

Figure 5. $\left|G_{M}^{n}\left(Q^{2}\right)\right|$ as measured by FENICE, compared to PQCD expectations [34] (dashed line) and as suggested by the average angular distribution (solid line).

unexpected and spectacular features also for time-like $Q_{p \bar{p}}^{2}$. BaBar has measured with unprecedented accuracy, by means of ISR, the $e^{+} e^{-} \rightarrow p \bar{p}$ cross section from the threshold up to $Q_{p \bar{p}}^{2} \sim 20 \mathrm{GeV}^{2} / \mathrm{c}^{4}$, finding out unexpected drops in the cross section at $2.2 \mathrm{GeV}$ and $2.9 \mathrm{GeV}$ as well as confirming a sharp drop of the magnetic form factor at threshold. Evidence for a ratio $\left|G_{E}^{p} / G_{M}^{p}\right|>1$ has also been found just above the threshold at odd with previous measurements at LEAR, but consistent with analyticity expectation [9]. The neutron time-like FF were poorly measured, only once, nevertheless showing unexpected features.

\section{Acknowledgments}

I warmly acknowledge the Institute for High Energy Physics of the Chinese Academy of Sciences in Beijing and the BaBar collaboration for having the opportunity to present this still challenging topic and S. Pacetti, E. P. Solodov and A. Zallo for their inestimable and friendly collaboration.

\section{References}

1. M.K. Jones et al., Phys. Rev. Lett. 84, 1398 (2000); O. Gayou et al., Phys. Rev. Lett. 88, 092301 (2002).

2. T. Massam, A. Zichichi, Nuovo Cim. A43, 1137 (1966).

3. F. Iachello, A.D. Jackson, A. Lande, Phys. Lett. B43, 191 (1973).

4. BaBar collaboration, Phys. Rev. D73, 012005 (2006).

5. A. Antonelli et al., Nucl. Phys. B517, 3 (1998).

6. S.D. Drell, F. Zachariasen, Electromagnetic Structure of Nucleon, Oxford University Press (1960).

7. S.J. Brodsky, G.R. Farrar, Phys. Rev. Lett. 31, 1153 (1973).

8. R.C. Walker et al., Phys. Rev. D49, 5671 (1994).

9. R. Baldini et al. Eur. Phys. J.C46, 421 (2006).

10. P.G. Blunden, W. Melnitchouk, J.A. Tjon, Phys. Rev. Lett. 91, 142303 (2003).

11. J. Arrington, Phys. Rev. C68, 034325 (2003).

12. R. Baldini, et al., Eur. Phys. J. C11, 709 (1999).

13. H. Czuz et al., Eur. Phys. J. C35, 527 (2004). 
14. L.D. Landau, E.M. Lifshits, Relativistic Quantum Theory, Mir (1975).

15. M. Conversi, T. Massam, T. Muller, A. Zichichi, Nuovo Cim. 40, 690 (1965).

16. G. Bardin et al., Nucl. Phys. B411, 3 (1994).

17. T.A. Armstrong et al., Phys. Rev. Lett. 70, 1212 (1993); M. Ambrogiani et al., Phys. Rev. D60, 032002 (1999); M. Andreotti et al., Phys. Lett. B559, 20 (2003).

18. M. Castellano et al., Nuovo Cim. A14, 1 (1973).

19. B. Delcourt et al., Phys. Lett. B86, 395 (1979).

20. D. Bisello et al., Nucl. Phys. B224, 379 (1983); Z. Phys. C48, 23 (1990).

21. G. Lepage, S. Brodsky, Phys. Rev. Lett. 43, 545 (1979).

22. BaBar Collaboration, B. Aubert et al., Nucl. Instr. And Meth. A479, 1 (2002).

23. H. Czyz, J.H. Khun, Eur. Phys. J. C18, 497 (2001).

24. M. Caffo, H. Czyz, E. Remiddi, Nuovo Cim. A110, 515 (1997); Phys. Lett. B327, 369 (1994).

25. E. Fermi, C.N. Yang, Phys. Rev. 76, 1739 (1949); S. Shapiro, Phys. Rep. 35, 129 (1978); C.B. Dover, M. Goldhaber, Phys. Rev. D15, 1997 (1977); J. M. Richard, Nucl. Phys. Proc. Suppl. 86, 361 (2000); A. Datta, P.J. O’ Donnel, Phys. Lett. B567, 273 (2003).

26. BESII collaboration, Phys. Rev. Lett. 91, 022001 (2003).

27. S. Pacetti, eConf C0309101, FRWP013 (2003); P.J. Franzini, F.J. Gilman, Phys. Rev. D32, 237 (1985).

28. R. Baldini et al., reported at the "Fenice" Workshop, Frascati (1988).

29. P.L. Frabetti et al., Phys. Lett. B514, 140 (2001); Phys. Lett. B578, 290 (2004).

30. BaBar collaboration, Phys. Rev. D73, 052003 (2006).

31. M. Agnello et al., Phys. Lett. B527 (2002) 39.

32. A. Sibirtsev, U.-G. Meissner et al., Phys. Rev. D71 054010 (2005).

33. S. Brodsky, Nucleon05 Frascati (2005).

34. H.W. Hammer, U.G. Meissner, Eur. Phys. J. A20, 469 (2004).

35. J.R. Ellis, M. Karliner, New J. Phys. 4, 18 (2002).

36. P. Cesselli, M. Nigro, C. Voci, Proc. Physics at Lear, Erice 11, 465 (1999). 